\title{
A Precise, Reduced-Parameter Model of Thin Film Electrolyte Impedance
}

\author{
Benjamin E. McNealy, ${ }^{\mathrm{a}, \mathrm{z}}$ Jun Jiang, ${ }^{\mathrm{b}}$ and Joshua L. Hertz ${ }^{\mathrm{a}, \mathrm{b}, *}$ \\ ${ }^{a}$ Department of Mechanical Engineering, University of Delaware, Newark, Delaware 19716, USA \\ ${ }^{b}$ Department of Materials Science and Engineering, University of Delaware, Newark, Delaware 19716, USA
}

\begin{abstract}
The extreme shape factors inherent in characterizing thin film electrolytes can present a challenge to quantitative interpretation of impedance spectra. Here, the impedance of a thin film ceramic electrolyte with surface microelectrodes is modeled via direct numerical solution of current conservation. Faradaic and non-faradaic currents at the electrode-electrolyte interface are modeled phenomenologically using a formulation based on the Butler-Volmer equation. The model is able to reproduce complex, experimentally obtained impedance spectra for Pt/YSZ and Pt/GDC cells using only four adjustable, physically intuitive parameters: electrolyte conductivity, permittivity, exchange current density, and double layer capacitance. Equivalent circuit models typically used to fit these spectra instead require six or more adjustable parameters with ambiguous physical meaning. Notably, the model described here is able to capture a heretofore unexplained intermediate frequency arc seen in the experimental results. A parametric study enables the mechanism of the intermediate frequency feature to be identified as a spreading resistance in the electrolyte that vanishes at high frequencies due to low-impedance dielectric transport of current across the electrode-electrolyte interface. The fitting results are validated by comparison of the parameter values with literature reports.

(C) The Author(s) 2015. Published by ECS. This is an open access article distributed under the terms of the Creative Commons Attribution 4.0 License (CC BY, http://creativecommons.org/licenses/by/4.0/), which permits unrestricted reuse of the work in any medium, provided the original work is properly cited. [DOI: 10.1149/2.0281506jes] All rights reserved.
\end{abstract}

Manuscript submitted January 12, 2015; revised manuscript received February 16, 2015. Published March 7, 2015.

Electrochemical impedance spectroscopy (EIS) is a commonly used experimental technique that can deconvolute the contributions of multiple physical processes to the overall electrical impedance of a sample. ${ }^{1}$ By fitting the experimental impedance spectrum with an appropriate physical model, the relevant properties of the system can be quantified. However, the choice of model is nontrivial and requires careful justification as any number of models may fit the EIS data equally well. ${ }^{2}$ An especially common way to model EIS data is to use an equivalent circuit, ${ }^{2-5}$ which lumps system properties into a network of resistors, capacitors, and other electrical circuit elements. While potentially useful, these models are also quite reductive and the physical meaning of the circuit elements can be ambiguous or otherwise prone to misinterpretation., ${ }^{5,6}$ To address these shortcomings, some researchers have instead developed more sophisticated physicochemical impedance models ${ }^{6-10}$ which are capable of directly relating the impedance to physical properties of the system.

Microelectrodes ${ }^{11}$ have various applications in electrochemistry, and in recent years have been increasingly used in the characterization of oxide ion conducting ceramics. ${ }^{12-16}$ These materials may be used in solid oxide fuel cells (SOFCs) and various other applications. Microelectrodes are generally used in fundamental electrochemical studies, where researchers prefer model systems with well-defined electrode and interfacial geometries. Non-porous point or pattern microelectrodes have the requisite geometric characteristics and can be fabricated in a straightforward fashion using thin film processes. A schematic of an electrochemical cell consisting of interdigitated microelectrodes patterned onto a ceramic electrolyte layer is shown in Fig. 1. The simple geometry of the electrodes allows the electrodeelectrolyte contact area and triple phase boundary (TPB) length to be easily quantified. ${ }^{12,17}$

In impedance studies of such cells, an anomalous intermediate frequency feature ${ }^{18,19}$ is sometimes observed. An example of such an impedance spectrum, obtained for a gadolinium doped ceria (GDC) thin film electrolyte with dense platinum electrodes at $652^{\circ} \mathrm{C}$, is shown in Fig. 2. The spectrum consists of three arcs, labeled I, II, and III in the figure. The high frequency arc I is typically attributed to the bulk electrolyte, while the low frequency arc III is attributed to the electrode reaction. However, the meaning of the intermediate frequency arc II is less clear. Hertz et al. attributed the intermediate frequency arc observed in platinum/yttria stabilized zirconia (YSZ) cells to a current

\footnotetext{
*Electrochemical Society Active Member.
}

zE-mail: bmcnealy@udel.ed constriction effect near the TPB. ${ }^{18}$ A schematic of the low and high frequency current distributions in the electrolyte is shown in Fig. 3a. Under DC or low frequency conditions, current is confined near the TPB, which is the only location where the oxygen vacancies, electrons, and oxygen gas required for the electrode reaction are all present. At higher frequency, displacement current is able to travel over the entire electrode-electrolyte interface and, according to this model, the constriction resistance vanishes. However, the researchers' quasi-DC numerical modeling approach predicted that an unrealistically small TPB size would be required to account for the magnitude of resistance observed experimentally.

The work described in this paper suggests instead that the mechanism for the intermediate frequency impedance is the spreading of current in the electrolyte at low frequencies. A spreading resistance refers to the increased resistance due to nonparallel current flow through a conductor. A thin film electrolyte considered alone has negligible spreading resistance. ${ }^{20}$ However, Zhang et al. have shown that contact between two phases with different conductivities can result in a much larger spreading resistance. ${ }^{21}$ A result similar to Zhang's could be expected if there is substantial resistance to current flow at the electrode-electrolyte interface. In this case, current is forced to spread across the whole electrode-electrolyte interface at low frequency. At higher frequencies, the interfacial impedance vanishes since the parallel capacitance of the interface allows current to cross the interface dielectrically. The current is thus able to take a shorter path through the electrolyte on average. The low and high frequency current paths through the electrolyte predicted by this hypothesis are shown in Fig. 3b.

It is also worth noting that the shape of the intermediate frequency feature shown in Fig. 2 is reminiscent of a Warburg impedance, which manifests as a 45-degree line in the impedance plane and often arises from a diffusion process. Although the impedance feature observed here is not strictly linear and has a "slope" closer to 30 degrees, the possibility that the intermediate frequency impedance could be due to diffusion still merits some discussion. As the experiments discussed in Refs. 18 and 19 were performed under atmospheric conditions, it is highly unlikely that the electrode reaction could be limited by gasphase diffusion at the frequencies under consideration. It is also known that bulk oxygen diffusion ${ }^{22}$ or diffusion-limited oxide formation ${ }^{23}$ can occur in the platinum electrode. However, the published diffusion coefficients ${ }^{23,24}$ are on the order of $10^{-23}$ to $10^{-22} \mathrm{~m}^{2} / \mathrm{s}$, too low to result in a characteristic frequency in the relevant range. Therefore, the possibility of a diffusion-related feature in the impedance spectrum was discounted. 


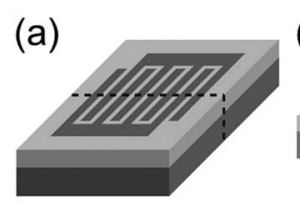

(b)

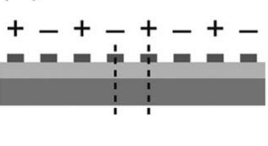

(d)

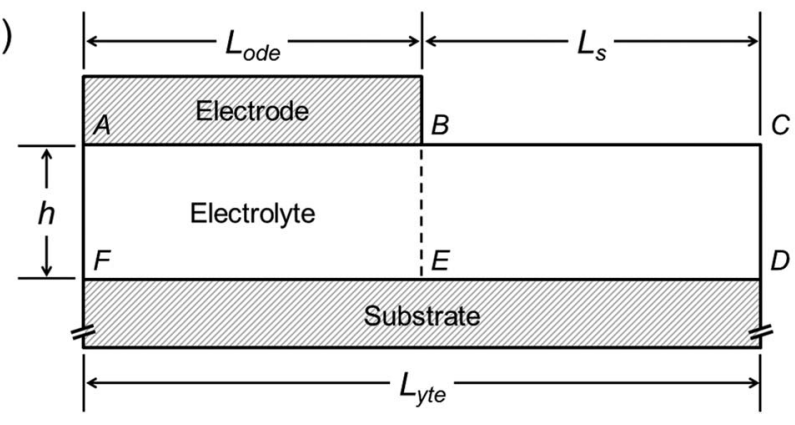

Figure 1. Schematic of a thin film electrochemical cell with interdigitated microelectrodes. The 3D geometry (a) can be reduced to the 2D geometry (d) as shown by slicing along the dashed lines shown in parts (a), (b), and (c).

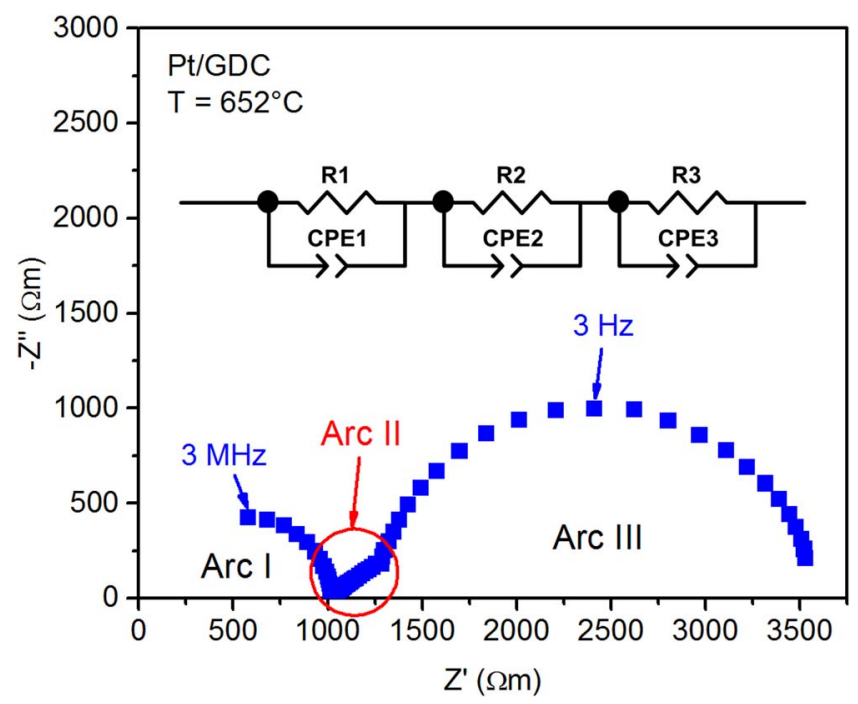

Figure 2. Sample impedance spectrum for a Pt/GDC specimen with an electrolyte thickness of $30 \mathrm{~nm}$ and electrode spacing and width of $25 \mu \mathrm{m}$. A possible equivalent circuit to fit the spectrum is shown in the inset.

In a conventional analysis, the impedance spectrum of Fig. 2 might be fitted with an equivalent circuit like the one shown inset in the figure, with a parallel R-CPE element corresponding to the intermediate frequency arc II. Such a model is capable of fitting the impedance spectrum with a high degree of fidelity, but it is difficult to ascribe a

(a)

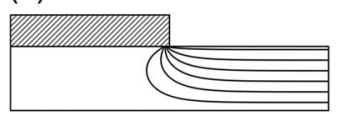

(b)

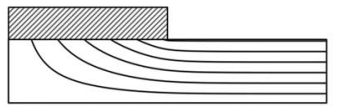

Low frequency
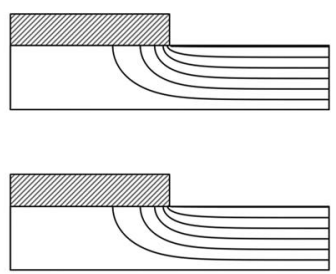

High frequency
Figure 3. Two proposed mechanisms for the intermediate frequency arc: (a) low frequency current constriction at TPB; (b) low frequency current spreading at electrode-electrolyte interface.
Table I. Properties of the Pt/YSZ and Pt/GDC samples.

\begin{tabular}{lll} 
& $\mathrm{Pt} / \mathrm{YSZ}$ & $\mathrm{Pt} / \mathrm{GDC}$ \\
\hline Electrode material & $\mathrm{Pt}$ & $\mathrm{Pt}$ \\
Electrode finger width & $25 \mu \mathrm{m}$ & $25 \mu \mathrm{m}$ \\
Electrode finger spacing & $25 \mu \mathrm{m}$ & $25 \mu \mathrm{m}$ \\
Electrolyte material & $\mathrm{Zr}_{0.91} \mathrm{Y}_{0.09} \mathrm{O}_{1.95}$ & $\mathrm{Ce}_{0.8} \mathrm{Gd}_{0.2} \mathrm{O}_{1.9}$ \\
Electrolyte thickness & $100 \mathrm{~nm}$ & $30 \mathrm{~nm}$ \\
Substrate & Amorphous $\mathrm{SiO}_{2}$ & Single crystal $\mathrm{Al}_{2} \mathrm{O}_{3}(0001)$
\end{tabular}

physical meaning to the arc II circuit parameters. In the present work, a continuum physical model is instead implemented using finite element software in order to simulate the electrochemistry of thin-film YSZ and GDC electrolytes with platinum surface microelectrodes. Our goal is to understand the physical origin of the measured impedance across all frequencies. In particular, we seek to understand the intermediate frequency arc and to improve the quantitative information that can be obtained from fitting of experimental results. Note that we do not seek with this work to explain the atomic mechanisms of phenomena such as double layer capacitance or polarization resistance. The model includes very basic geometrical parameters, material properties, and boundary conditions, but no assumptions regarding the current path (e.g. Fig. 3 a vs. 3b) are made.

\section{Methods}

Experimental.-Data from previously reported experimental work on thin film Pt/YSZ and Pt/GDC electrochemical cells ${ }^{18,19}$ were used for validation of the present modeling work. The samples were fabricated by patterning dense, interdigitated platinum microelectrodes onto sputtered YSZ and GDC films using photolithography. While the samples measured in Refs. 18 and 19 are similar in geometry and materials set, it should be noted that they were created and measured in different labs and on different equipment. The details of the fabrication and impedance characterization processes used in these studies have been reported elsewhere. ${ }^{17,19}$ The sample properties relevant to the present work are given in Table I. Impedance spectra were collected for each sample in air. The YSZ specimens were measured using a Solartron 1260 impedance analyzer over a frequency range of $0.01-10^{7} \mathrm{~Hz}$ at $333-394^{\circ} \mathrm{C}$ with a signal amplitude of $20 \mathrm{mV}$, while the GDC specimens were measured using a Novocontrol Alpha impedance analyzer over a frequency range of $0.01-3 \times 10^{6} \mathrm{~Hz}$ at $401-652^{\circ} \mathrm{C}$ with a signal amplitude of $1 \mathrm{mV}$.

Model setup.- The electrochemical cell to be modeled is shown schematically in Fig. 1a. The geometry consists of a thin, solid electrolyte film deposited on a chemically inert substrate, with interdigitated platinum electrodes patterned on the top surface of the electrolyte. An AC potential is applied between the electrodes. Assuming the electrode fingers are long enough for edge effects to be negligible, the geometry can be simplified to two dimensions by considering the cross-sectional plane indicated in Fig. 1b. Making use of mirror and translational symmetries in the sample geometry, the model domain can be further simplified as shown in Fig. 1c. Finally, as the potentials applied to the two electrodes are equal in magnitude but opposite in polarity, the antisymmetry of the sample may be taken advantage of by bisecting the model domain to obtain the geometry shown in Fig. 1d. It is evident by inspection that the potential along the right border $(C D)$ is zero. The simplified two-dimensional model geometry is shown in Fig. 4. While the substrate is considered to have negligible DC conductivity compared to the electrolyte film, it may still provide non-negligible dielectric conduction. Therefore the model includes a portion of the substrate in addition to the electrode and electrolyte.

The impedance of the system is determined by solving for the current everywhere in the domain following a similar approach to previous work by Fleig and Maier ${ }^{25}$ as well as the authors. ${ }^{26}$ The model presented in Ref. 26, which considered only the electrolyte, 


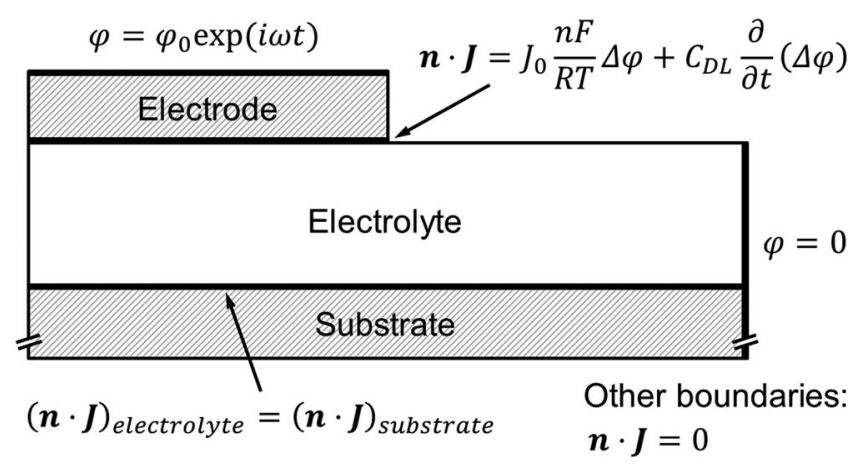

Figure 4. Model boundary conditions. All unlabeled boundaries obey the zero current condition given by Eq. 6 .

has been extended here to include also the impedance associated with the electrode-electrolyte interface. In addition, the earlier modeling approach has been modified by solving for current directly in order to facilitate the use of boundary conditions given explicitly in terms of current. In the absence of any current sources or sinks, the current density $J$ in the model domain is governed by

$$
\nabla \bullet J=0
$$

Further,

$$
J=-\kappa \nabla \varphi
$$

where $\varphi$ is the electric potential. The complex conductivity $\kappa$ is defined as

$$
\kappa=\sigma+i \omega \varepsilon \varepsilon_{0}
$$

where $\sigma$ is conductivity, $\varepsilon$ is relative permittivity, $\varepsilon_{0}$ is the permittivity of free space, and $\omega$ is angular frequency.

The boundary conditions used in the model are shown in Fig. 4. An AC potential

$$
\varphi=\varphi_{0} \exp (i \omega t)
$$

is applied at the electrode surface. At $x=\mathrm{L}$, the aforementioned symmetry condition

$$
\varphi=0
$$

is applied. All other exterior boundaries were considered to be insulating to current flow with

$$
\vec{n} \bullet J=0
$$

where $\vec{n}$ is a unit vector normal to the surface. As a consequence of the above formulation, both $\varphi$ and $J$ are complex quantities.

Difficulty arises in the modeling of the electrode kinetics, since even for model systems (e.g. Pt/ $\mathrm{O}_{2} / \mathrm{YSZ}$ ) the electrochemical reactions involved are not yet completely understood. ${ }^{27}$ However, studies of platinum electrodes on solid oxide electrolytes ${ }^{28}$ have demonstrated that the current-overpotential relationship in such electrodes can be modeled using the well-known Butler-Volmer equation from classical aqueous electrochemistry. Later work has shown that the electrode polarization is controlled largely or entirely by processes other than electrochemical charge transfer, ${ }^{27,29,30}$ and thus the physical basis for Butler-Volmer kinetics is lacking. Nevertheless, the mathematics remain useful phenomenologically, and researchers have compiled values of the exchange current density for $\mathrm{Pt} / \mathrm{O}_{2} / \mathrm{YSZ}$ and other systems under various experimental conditions. ${ }^{30}$ As the focus of the present work is not a detailed microscopic understanding of the electrode kinetics, a phenomenological approach will suffice. Therefore the electrode-electrolyte interface will be modeled using Butler-Volmer kinetics, and the exchange current density treated as a strictly empirical parameter.

EIS experiments are generally performed with a small signal amplitude $\varphi_{0}$ in order to remain in the linear range of the I-V curve, so the linearized form of the Butler-Volmer equation ${ }^{31}$

$$
J=J_{0} \frac{n F}{R T} \eta
$$

is appropriate to the present case. In this equation $J_{0}$ is the exchange current density, $n$ is the charge number, $F$ is the Faraday constant, $R$ is the molar gas constant, $T$ is absolute temperature, and $\eta$ is the overpotential. The charge transfer coefficient $\alpha$ does not appear in the linearized form of the equation as the I-V relationship is independent of $\alpha$ in the low-overpotential region. ${ }^{31}$ Due to the symmetry of the electrochemical cell, the equilibrium potential of the electrodes can be neglected and $\eta$ taken as simply the potential difference $\Delta \varphi$ across the interface. The temperature $T$ was treated as a constant parameter, assuming the sample is thermally equilibrated and neglecting any Joule heating due to current flux. The latter assumption was considered to be reasonable for the geometry considered here as the electrical potentials involved are only in the millivolt range and the volume of the electrolyte is very small relative to its surface area, allowing for effective cooling. Further, the maximum current flux occurs in a region very near the free surface of the electrolyte (see Fig. 3), enabling the heat generated to dissipate easily. This was further justified using a simple DC electrostatic/heat transfer model of the electrolyte, which predicted negligible temperature change for YSZ even under worst case conditions (poor convection at the free surface and complete thermal insulation at all other boundaries including the electrolytesubstrate interface).

In addition to the faradaic current flux represented by Eq. 7, displacement current across the interface was considered by incorporating a double layer capacitance with current density

$$
J=C_{D L} \frac{\partial \eta}{\partial t}
$$

The parameter $C_{D L}$ should be thought of as a phenomenological descriptor of the capacitive properties of the electrode-electrolyte interface rather than a literal double layer capacitance, as the capacitance of the platinum/solid oxide interface is thought to arise from chemical processes rather than a classical double layer mechanism ${ }^{23,27,32} \mathrm{Nev}$ ertheless, a phenomenological treatment is adequate for the present work. Combining Eqs. 7 and 8, the boundary condition at the electrode-electrolyte interface can be expressed as

$$
\vec{n} \bullet J=J_{0} \frac{n F}{R T} \Delta \varphi+C_{D L} \frac{\partial}{\partial t}(\Delta \varphi)
$$

At the interface between the electrolyte and substrate, the model requires continuity of the normal component of the complex current density

$$
(\vec{n} \bullet J)_{\text {electrolyte }}=(\vec{n} \bullet J)_{\text {substrate }}
$$

The substrate is given negligible DC conductivity but finite permittivity allowing for capacitive currents.

For any single value of frequency, the time- and space-dependent current and potential distributions are determined by solving Eq. 1 subject to the boundary conditions Eqs. 4, 5, 6, 9, and 10, and the initial condition $\varphi=0$ everywhere in the domain. The solution was obtained numerically using COMSOL Multiphysics (COMSOL AB, v. 4.4). After determining the current distribution, the total current through the specimen can be calculated by integrating the complex current density over any equipotential surface, the most convenient of which is the antisymmetry boundary $x=\mathrm{L}$, where $\varphi=0$.

$$
I=\int_{s y m} J \cdot d s
$$

The complex impedance $Z$ of the specimen ${ }^{25}$ can then be determined from

$$
Z=\varphi_{0} / I
$$

Due to the two-dimensional nature of the system, $Z$ here has units of $\Omega \cdot \mathrm{m}$. The value of $Z$ was calculated at time $t=2 / f$, i.e. after 
two AC cycles. It was determined that this was sufficient to limit any transient fluctuations in the magnitude of $Z$ to less than $0.01 \%$. The full impedance spectrum was then obtained by repeating the above process over a range of frequency values. The frequencies of interest typically ranged from $\mathrm{mHz}$ to $\mathrm{MHz}$ with 10 data points per frequency decade. In order to accommodate this wide frequency range, the time step used in each simulation was adjusted to maintain an equal time steps per AC cycle regardless of frequency. It was determined that 10 time steps per cycle was sufficient to accurately calculate the impedance.

Mesh.- In order to balance solution accuracy with computation time, proper mesh selection was important. For the present study, a quadrilateral mesh with 17,500 elements was selected. The elements were mapped so that the mesh was much finer in the electrode and electrolyte layers than in the substrate. By performing a mesh convergence study, the maximum error in the calculated impedance magnitude (after two AC cycles) using the mapped mesh was estimated at $0.01 \%$ at $0.01 \mathrm{~Hz}$ and $1 \%$ at $300 \mathrm{MHz}$.

Fitting procedure.- One of the key purposes of the finite element modeling performed here was comparison of the resulting calculated impedance spectra to previously measured experimental spectra. This procedure is sometimes complicated by experimental data generally suffering from poorer resolution at measurement frequencies below approximately $0.1 \mathrm{~Hz}$. In such cases, a least-squares fitting procedure can yield a poor estimate of the low frequency arc parameters. Instead, the fitting was performed graphically in order to maintain equal radii between the low frequency arc in the experimental spectrum and the fitted model results. The fitting procedure allowed the parameter values $\varepsilon$ and $C_{D L}$ to be estimated with a resolution of \pm 0.1 (dimensionless) and $\pm 1 \mu \mathrm{F} / \mathrm{cm}^{2}$, respectively. The values of $\sigma$ and $J_{0}$ derived from the fitting procedure were within $1 \%$ of the values derived from an equivalent circuit fit to the same experimental data, demonstrating the robustness of the graphical fitting procedure.

\section{Results and Discussion}

Sample results. - As an example of the model output, a simulated impedance spectrum for a $25 \mathrm{~nm}$ thick electrolyte is shown in Fig. 5. As the parameter values used in this illustrative simulation were selected arbitrarily, the model output is not expected to quantitatively match the experimental data in Fig. 2 (quantitative fitting

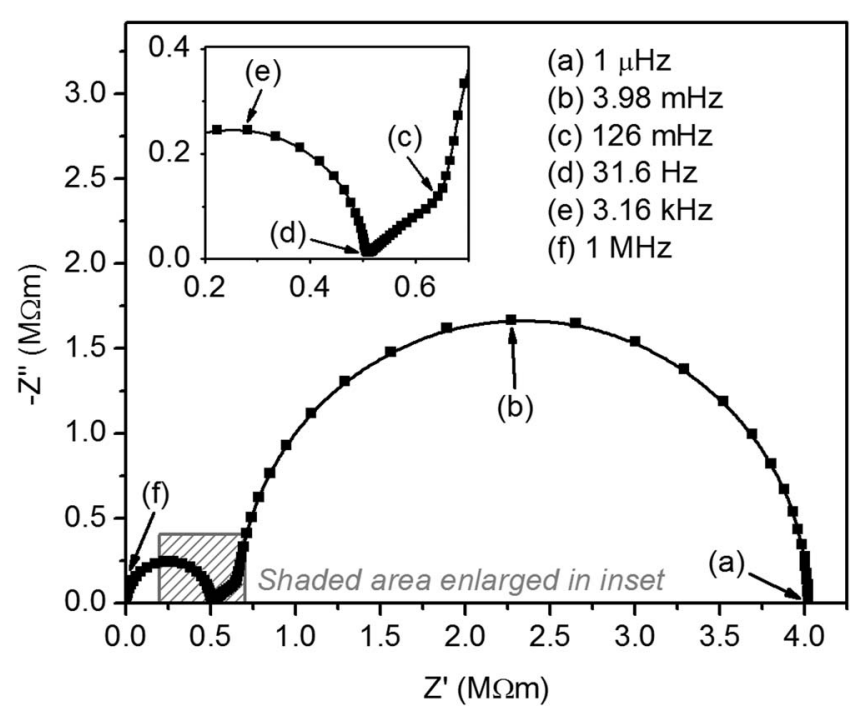

Figure 5. Impedance response predicted by the model for a $25 \mathrm{~nm}$ film at $700^{\circ} \mathrm{C}$ with $L_{\text {ode }}=L_{s}=12.5 \mu \mathrm{m}, \sigma=10^{-5} \mathrm{~S} / \mathrm{cm}, J_{0}=10^{-4} \mathrm{~mA} / \mathrm{cm}^{2}, C_{D L}$ $=100 \mu \mathrm{F} / \mathrm{cm}^{2}$, and $\varepsilon=10$. The intermediate frequency region is enlarged in the inset. The frequencies of a few key points are indicated.
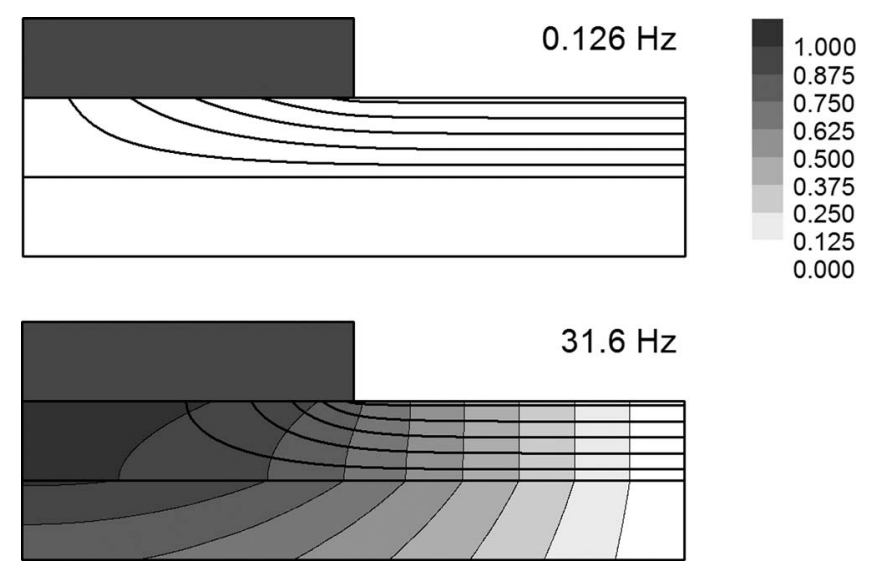

Figure 6. Contours of electrical potential in $\mathrm{mV}$, indicated by black and white color scale, in the electrode, electrolyte, and substrate at $f=0.126 \mathrm{~Hz}$ and $31.6 \mathrm{~Hz}$. The vertical dimension has been exaggerated and the substrate truncated to aid visualization. The distribution of current in the electrolyte is also shown via solid lines.

of the experimental results will be discussed later in this section). Still, the qualitative similarity is striking. Three separate impedance features can be distinguished, including a depressed partial arc in the intermediate frequency region.

In order to clarify the origin of the intermediate frequency arc, the current and potential distributions in the relevant frequency range were examined. Fig. 6 shows the distributions at $f=0.126 \mathrm{~Hz}$ and $31.6 \mathrm{~Hz}$, corresponding to the low- and high-frequency endpoints, respectively, of the intermediate frequency arc. The figure reveals that at $0.126 \mathrm{~Hz}$ or below, nearly all of the potential drop in the specimen occurs at the electrode-electrolyte interface, and the current distribution along the length of this interface is nearly uniform. At $31.6 \mathrm{~Hz}$ or higher, the potential drop across the interface is small compared to that in the electrolyte. At these relatively high frequencies, current is no longer distributed over the entire interface and instead travels through a narrower region near the edge of the electrode.

This frequency-dependent change of current path through the electrolyte is due to low-impedance dielectric current flux across the electrode-electrolyte interface. At relatively low frequencies, the impedance of the interface is large compared to that of the electrolyte and current travels further on average through the electrolyte to spread out over the entire interface. At higher frequencies, the interfacial impedance begins to vanish due to non-negligible doublelayer displacement current, and current is able to travel a shorter path that bypasses a large portion of the electrolyte. Thus the intermediate frequency impedance arc is associated with a kind of spreading resistance arising from the sample geometry and relative impedances of the electrolyte and the electrolyte-electrode interface. Specifically, it is related to the portion of the electrolyte which is covered by the electrode finger.

Fitting. - The model was validated by comparing the calculated impedance spectra to previously reported experimental impedance measurements of YSZ and GDC thin film samples with interdigitated platinum electrodes. ${ }^{18,19}$ The calculated spectra were fitted to the experimental data by adjusting the values of four parameters: electrolyte conductivity, permittivity, exchange current density, and double layer capacitance. All other model parameters, including geometric parameters and temperature, were fixed to the known values determined by the experimental conditions. An example result from this procedure for a $\mathrm{Pt} / \mathrm{GDC}$ specimen at $652^{\circ} \mathrm{C}$ is shown in Fig. 7. Although the experimental spectrum shows some depression of the low and high frequency arcs, the overall fit of the model is very good and all key features are captured. The ability to model the system with only four fitting parameters offers a notable advantage compared to a more conventional equivalent circuit. For example, the 

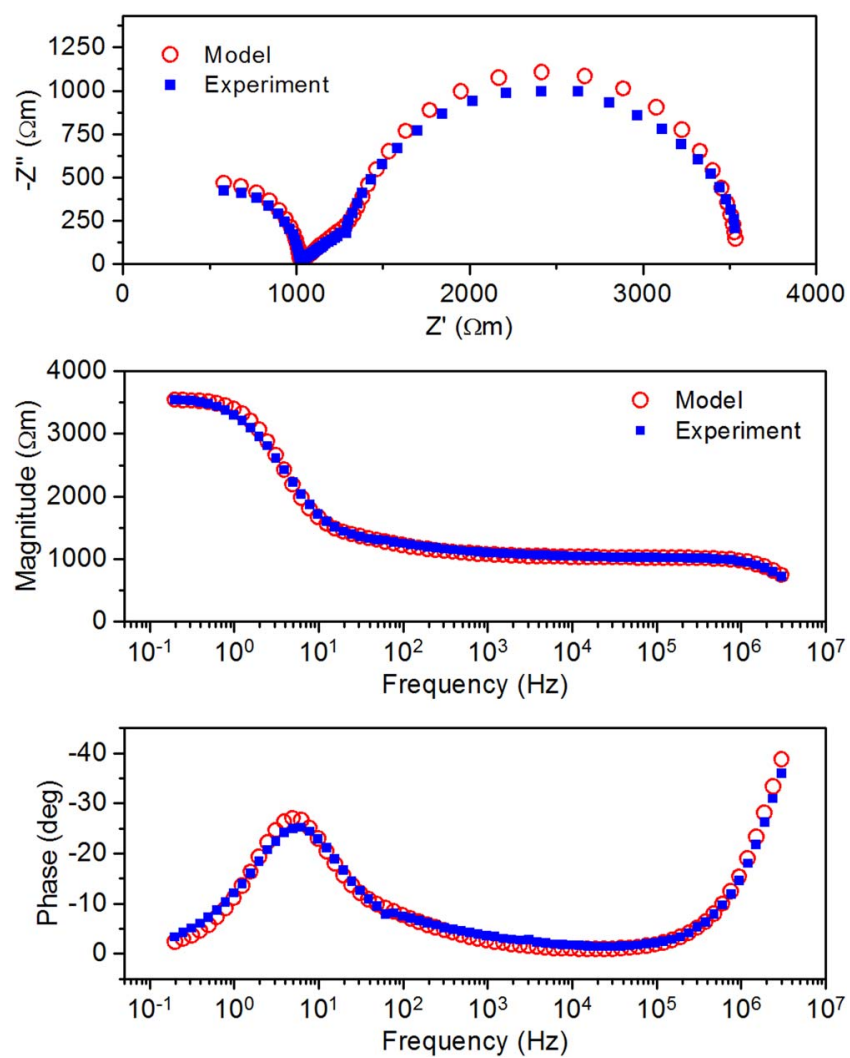

Figure 7. Bode and Nyquist plots of the impedance of a Pt/GDC specimen at $652^{\circ} \mathrm{C}$, along with the model fit.

equivalent circuit in Fig. 2 requires 9 fitting parameters. Furthermore, all four parameters determined by the model described in this work represent intuitively meaningful (if phenomenological) parameters of the system, which is not always the case for an equivalent circuit model.

After repeating the fitting routine for a range of samples and temperatures, the values determined for the conductivity of YSZ and GDC

\begin{tabular}{|c|c|c|c|c|c|}
\hline Material & Spec. no. & Dopant \% & Thickness (nm) & Substrate & Ref \\
\hline YSZ & 1 & 9.5 & 125 & $\mathrm{MgO}(001)$ & 37 \\
\hline YSZ & 2 & 9.5 & 210 & $\mathrm{MgO}(100)$ & 38 \\
\hline YSZ & 3 & 8.7 & 107 & $\mathrm{MgO}(110)$ & 39 \\
\hline YSZ & 4 & 9 & 100 & $\mathrm{Al}_{2} \mathrm{O}_{3}(0001)$ & 40 \\
\hline GDC & 1 & 20 & 110 & $\mathrm{Al}_{2} \mathrm{O}_{3}$ & 41 \\
\hline GDC & 2 & 20 & 170 & $\mathrm{MgO}(001)$ & 42 \\
\hline GDC & $3 a$ & 20 & 100 & $\mathrm{Al}_{2} \mathrm{O}_{3}$ & 34 \\
\hline GDC & $3 b$ & 20 & 100 & $\mathrm{SiO}_{2}$ & 34 \\
\hline GDC & $4 a$ & 21 & $500-1800$ & $\mathrm{Al}_{2} \mathrm{O}_{3}(0001)$ & 35 \\
\hline GDC & $4 b$ & 21 & $500-1800$ & $\mathrm{Pt}(111)$ & 35 \\
\hline
\end{tabular}

thin films were compared to literature values. As the reported conductivities of YSZ and GDC thin films can vary widely, a representative selection of films most similar to those considered in the present study was compiled. These samples are listed in Table II. The conductivities of representative bulk YSZ and GDC ceramics were also included. Since these measurements show a much smaller variation from sample to sample compared to the thin films, the bulk conductivities were reported as averages of several different samples. The bulk YSZ conductivity was obtained by linear regression of 9 different samples, compiled in Ref. 33. The conductivity of bulk GDC was obtained by linear regression of 3 different samples. ${ }^{34-36}$ The conductivity results are shown in Fig. 8. It is evident that both the conductivity magnitude and activation energy for the YSZ sample agree closely with literature results for similar specimens and for the bulk ceramic. The activation energy for the GDC sample is somewhat smaller than the published bulk and thin film values; however, there is considerable variation among the literature results. The magnitude of the GDC conductivity obtained from the model is slightly reduced compared to the bulk GDC results, but well within the range of reported values for thin films.

The second parameter obtained by fitting experimental data, the exchange current density, is shown in Fig. 9 along with several reported values of the same from the literature. ${ }^{1,29,43-45}$ The values obtained from the model for YSZ are slightly larger than the literature results when extrapolated to the lower measurement temperatures of
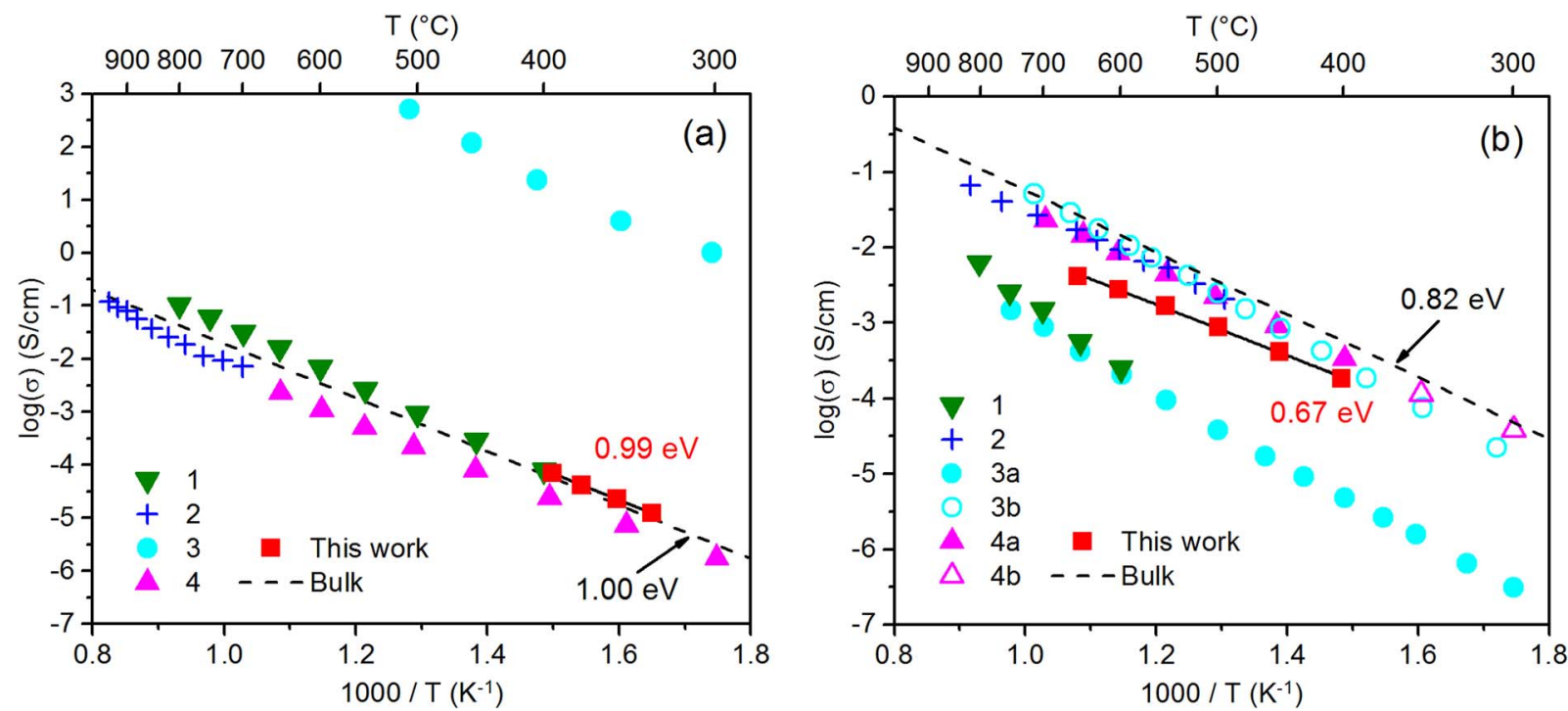

Figure 8. Arrhenius plot of electrolyte conductivity for (a) YSZ and (b) GDC thin films obtained by model fitting, compared to literature results for similar thin film specimens as well as bulk ceramics. The thin film specimens selected from the literature are compiled in Table II. The bulk conductivities were obtained by linear regression of several sets of data as explained in the text. The activation energy obtained from the model results, as well as the bulk activation energy, is indicated in each plot. 

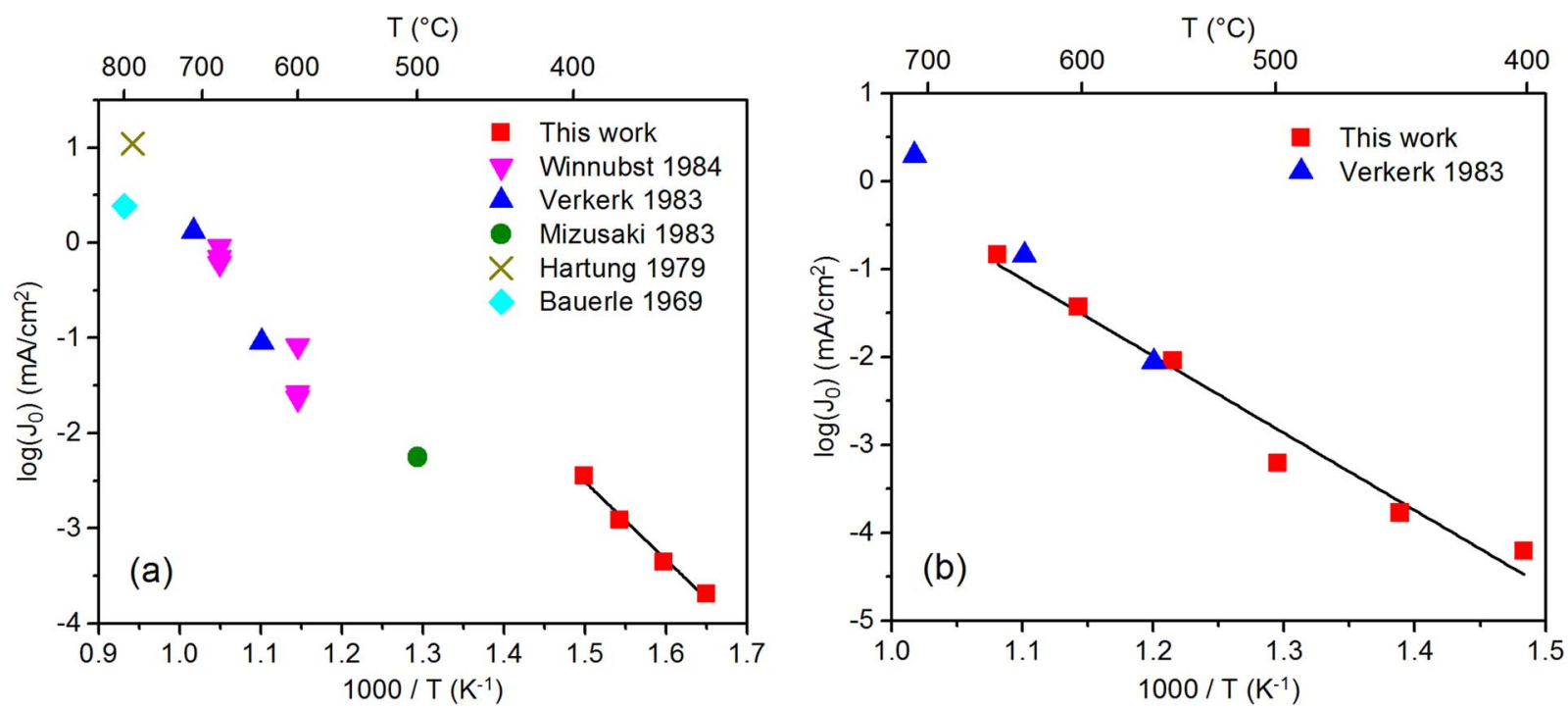

Figure 9. Arrhenius plot of exchange current density for Pt electrodes on (a) YSZ and (b) GDC. Model fit results from the present work are shown along with values reported previously in the literature.

the spectra fit in this work, but it bears mentioning that the reported values of $J_{0}$ vary by nearly an order of magnitude. Fewer values of $J_{0}$ are available for GDC, but the model agrees very well with the results from Ref. 29.

The third fitting parameter determined from the model's fit to the experimental data is the double layer capacitance. The values determined for the YSZ samples increased monotonically with temperature, ranging from $290 \mu \mathrm{F} / \mathrm{cm}^{2}$ at $333^{\circ} \mathrm{C}$ to $950 \mu \mathrm{F} / \mathrm{cm}^{2}$ at $394{ }^{\circ} \mathrm{C}$. This is consistent with values reported in the literature, which typically range from 60 to $550 \mu \mathrm{F} / \mathrm{cm}^{2}$ and occasionally higher. ${ }^{1,23,29,43}$ The literature values do not show a clear temperature dependence. As noted earlier, these values likely represent an electrochemical pseudocapacitance rather than a true double layer capacitance. The value of $C_{D L}$ for the GDC specimens varied from 80 to $325 \mu \mathrm{F} / \mathrm{cm}^{2}$ with no clear dependence on temperature, which also seems reasonable in light of the YSZ results.

The last of the four fitting parameters is the permittivity $\varepsilon$, which would intuitively be expected to represent the permittivity of the electrolyte film. However, the substrate permittivity was found to be far more significant to the overall impedance spectrum since the substrate thickness is so much larger than that of the electrolyte. In fact, a sensitivity study revealed that the impedance magnitude is more sensitive to the substrate permittivity than to that of the electrolyte unless the electrolyte is roughly the same thickness as the substrate or larger. As this was not the case in the present samples, the substrate permittivity rather than the electrolyte permittivity was chosen as the fitting parameter, and the electrolyte permittivity was assigned a constant value of 10 for all of the fitting. Adjusting this value within a reasonable range had very little observable effect on the results.

For the YSZ specimens, $\varepsilon$ varied from 8.3 to 8.8 and decreased slightly with increasing temperature. This is more than twice as large as the value of $\varepsilon \approx 3.8$ reported in the literature for fused silica, ${ }^{46}$ but still of a reasonable order of magnitude. The permittivity of the GDC specimens also generally decreased with increasing temperature. The values varied from 12.5 at $652^{\circ} \mathrm{C}$ to 24.6 at $447^{\circ} \mathrm{C}$ (the permittivity at $401^{\circ} \mathrm{C}$ was slightly lower at 23.1 ). For comparison, the expected permittivity of sapphire ${ }^{47,48}$ varies from $8.9-11.6$ depending on the crystal orientation. However, the sensitivity of the impedance to permittivity is quite low relative to the resolution of the fitting technique. Therefore, it appears that neither the film nor the substrate permittivity can be precisely determined using this technique. Stray capacitances in the experimental setup may be yielding anomalously high values in the model.
Parametric studies. - The intermediate frequency arc was further quantified via parametric study to determine when and why it forms a significant part of the overall measured impedance of the sample, and whether it can be of use in characterizing material properties of the electrode or electrolyte. The parameters of interest were the electrolyte thickness $h$, the electrolyte length $L_{y t e}$, the electrode finger width $L_{\text {ode }}$, the electrolyte conductivity $\sigma$, and the exchange current density $J_{0}$.

The effect of varying the electrolyte thickness is demonstrated in Fig. 10, which shows impedance spectra for four otherwise identical samples with different film thicknesses. As the high frequency impedance arc was not of interest here, it was cropped out of the figure and the real axis was re-zeroed at the high frequency extreme of the intermediate arc in order to enable direct quantitative comparison between the different cases. The magnitude of the intermediate frequency arc decreases with increasing thickness, which explains why this arc has only been reported for measurements on samples with nanoscale thickness; for $h=25 \mathrm{~nm}$ the intermediate arc is barely evident, whereas for $h=1 \mathrm{~nm}$ its magnitude is similar to that of the electrode arc. In addition, the apparent magnitude of the low frequency (electrode) arc also increases visibly for smaller values of $h$. This indicates that equivalent circuit fitting could yield inaccurate values of the electrode parameters in this case, an important finding that is discussed further in the next section. Note that the assumption of continuum physics is likely not completely valid at $h=1 \mathrm{~nm}$, but the results are included here for demonstration purposes.

In order to better compare these results, quasi-DC resistances were deconvoluted for the electrode, the electrolyte, and the intermediate frequency arc. The quasi-DC electrolyte resistance $R_{y t e}$ was obtained from the model by calculating the total impedance at very low frequency $\left(f=10^{-12} \mathrm{~Hz}\right)$ with $J_{0}$ sufficiently large to render the electrode contribution negligible $\left(J_{0}=10^{6} \mathrm{~mA} / \mathrm{cm}^{2}\right)$. The values so obtained agree closely with the theoretical electrolyte resistance, ${ }^{49}$ given by

$$
R_{y t e, t h e o}=\frac{L_{y t e}-L_{\text {ode }}}{h w \sigma}+R_{\text {spread }}
$$

where $w$ is the out-of-plane specimen width, taken as unity in the present work. The first term in Eq. 13 is simply the bulk resistance of the exposed portion of the electrolyte film (region $B C D E$ in Fig. 1), while $R_{\text {spread }}$ is the additional resistance incurred between plane $B E$ and the electrode. When $L_{\text {ode }} / h>2$, which is the case for most thin 

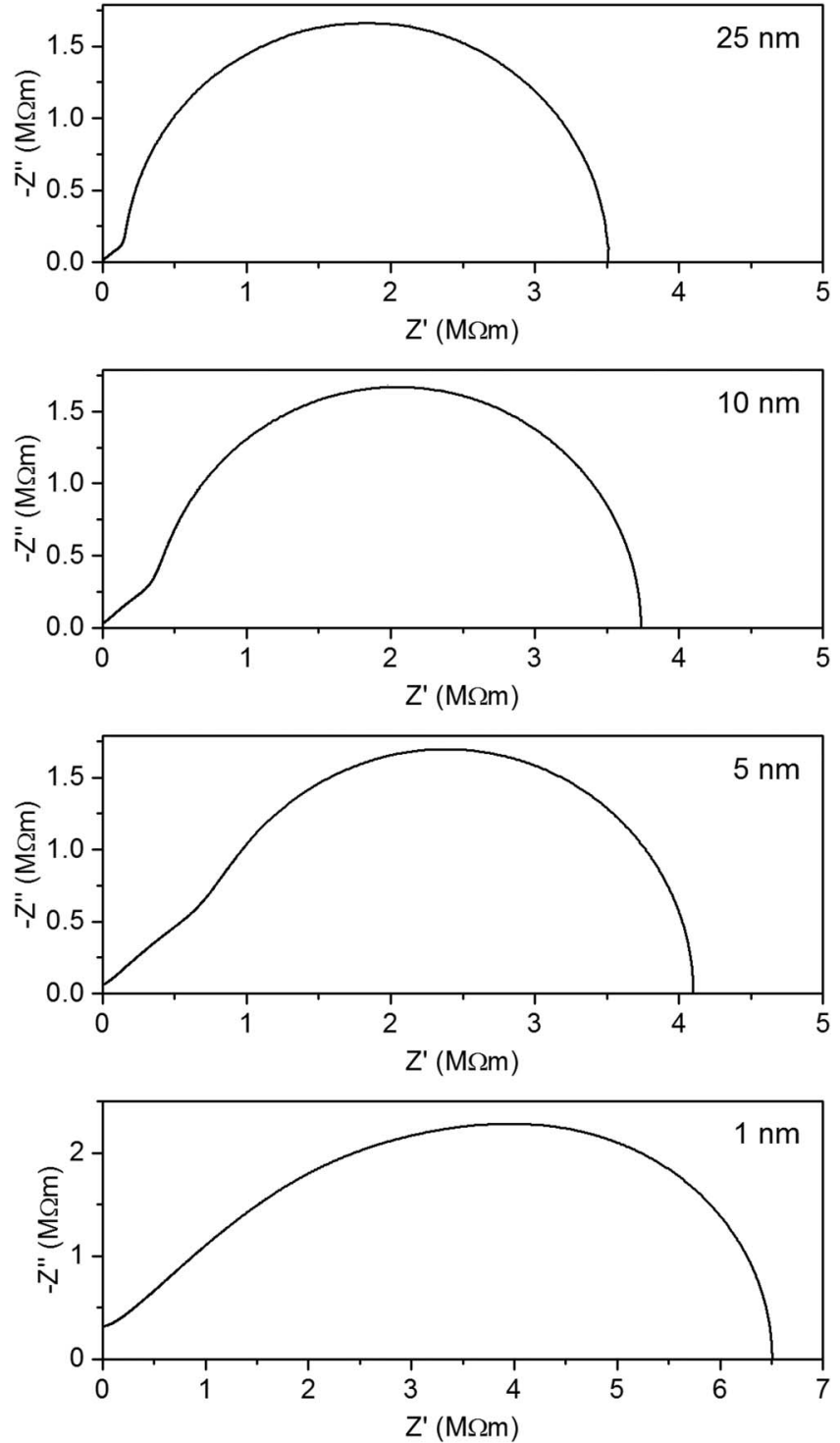

Figure 10. Low and intermediate frequency portions of the impedance spectra for specimens with the indicated electrolyte thickness and $L_{\text {ode }}=L_{s}=12.5$ $\mu \mathrm{m}$. The figure has been cropped and re-zeroed on the real axis in order to ignore the high frequency impedance arc. Note change of scale for the $1 \mathrm{~nm}$ specimen.

films, $R_{\text {spread }}$ can be approximated ${ }^{49}$ as

$$
R_{\text {spread }}=\frac{2 \ln 2}{\pi w \sigma} \approx \frac{0.441}{w \sigma}
$$

In most cases, $R_{\text {spread }}$ is negligible compared to the bulk resistance. It should be noted that the first term of Eq. 13 differs from the expression given in Ref. 49 by a factor of 2 owing to the choice of geometric repeat unit.

In a manner analogous to the procedure above, the quasi-DC resistance of the electrode, $R_{\text {ode }}$, was obtained from the model by calculating the total impedance at very low frequency $\left(f=10^{-12} \mathrm{~Hz}\right)$ with $\sigma$ sufficiently large that the impedance contribution of the electrolyte was negligible $\left(\sigma=10^{4} \mathrm{~S} / \mathrm{m}\right)$. As expected, the value of $R_{\text {ode }}$ so calculated agrees closely with the theoretical electrode resistance

$$
R_{\text {ode } \text { theo }}=\frac{1}{L_{\text {ode }} w} \cdot \frac{R T}{J_{0} n F}
$$

Finally, the effective resistance of the intermediate frequency arc, $R_{2}$, was quantified by subtracting $R_{y t e}$ and $R_{\text {ode }}$ from the total quasi-DC

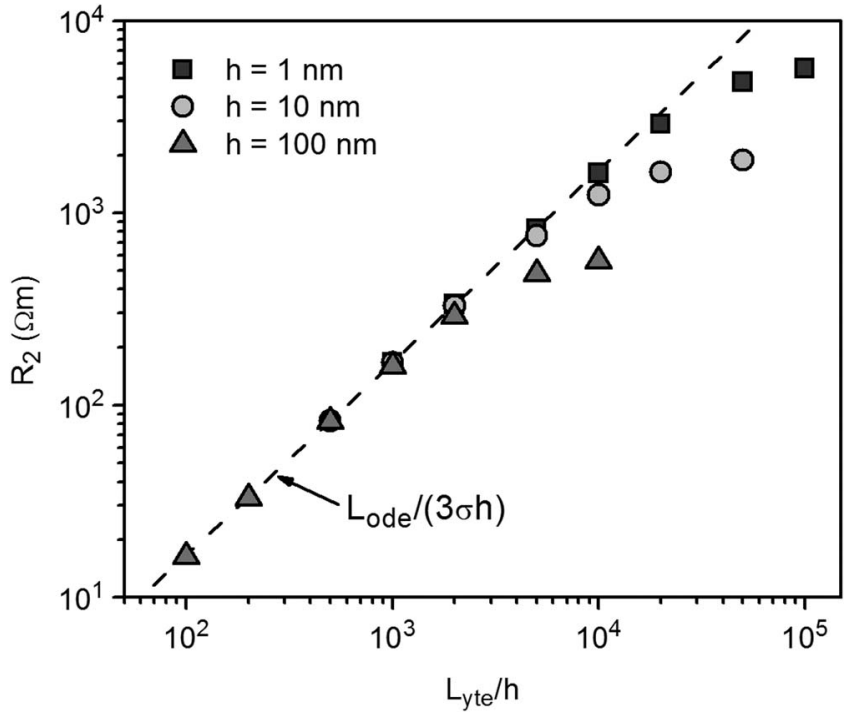

Figure 11. Resistance $R_{2}$ vs. $L_{y t e} / h$ for various values of $h$, with $\sigma=10^{-3}$ $\mathrm{S} / \mathrm{m}$ and $J_{0}=10^{-4} \mathrm{~mA} / \mathrm{cm}^{2}$. The electrode finger width and spacing are equal. The dashed line is a plot of the semi-empirical expression indicated.

resistance:

$$
R_{2}=R_{\text {total }}-R_{\text {yte }}-R_{\text {ode }}
$$

By inspection, the values derived using the above procedure for $R_{2}$, $R_{y t e}$, and $R_{\text {ode }}$ corresponded well to the approximate diameters of the (overlapping) arcs seen in the impedance spectra calculated for each model. The values of $R_{2}, R_{y t e}$, and $R_{\text {ode }}$ were compiled for a range of model geometries for further analysis.

The relationship between $R_{2}$ and the electrolyte aspect ratio $L_{y t e} / h$ is shown in Fig. 11. A linear trend corresponding to the semi-empirical equation

$$
R_{2}=\frac{L_{\text {ode }}}{3 \sigma h}
$$

is apparent for small values of $L_{y t e} / h$ (i.e., where the electrolyte is thick relative to the electrode spacing), but as the ratio is increased a deviation from linearity is observed. The onset of this deviation occurs at a lower value of $L_{y t e} / h$ for a larger electrolyte thickness, with a linear relationship observed only when $R_{\text {ode }} \gg R_{y t e}$. Based on this observation, Eqs. 13 and 15 can be combined (assuming negligible high frequency spreading resistance) to derive a condition for linearity. This yields

$$
\frac{h}{L_{\text {ode }}\left(L_{\text {yte }}-L_{\text {ode }}\right)} \frac{\sigma R T}{J_{0} n F} \gg 1
$$

For a given value of $L_{y t e}$, the product $\left(L_{y t e}-L_{o d e}\right) L_{o d e}$ is maximized when the electrode finger width and spacing are equal; i.e. $L_{o d e}=$ $0.5 L_{y t e}$. Keeping in mind that a lower bound on the quantity on the left-hand side of Eq. 18 is being sought, the inequality can then be rewritten as

$$
\frac{h \sigma R T}{L_{\text {ode }}{ }^{2} J_{0} n F} \gg 1
$$

which establishes the range of electrolyte aspect ratios for which $R_{2}$ vs. $L_{y t e} / h$ is linear. The dimensionless quantity on the left-hand side of Eq. 19 will be denoted $S$ for future reference.

The magnitude of the intermediate frequency impedance arc also depends on the electrode finger width and spacing. Impedance spectra for a few representative values of the coverage ratio $L_{\text {ode }} / L_{y t e}$ are shown in Fig. 12. The ratio varies from a limiting case of 0 as the electrode finger width approaches zero to 1 as the finger spacing approaches zero. A coverage ratio of 0.5 indicates that the finger width and spacing are equal. As can be observed from the figure, 


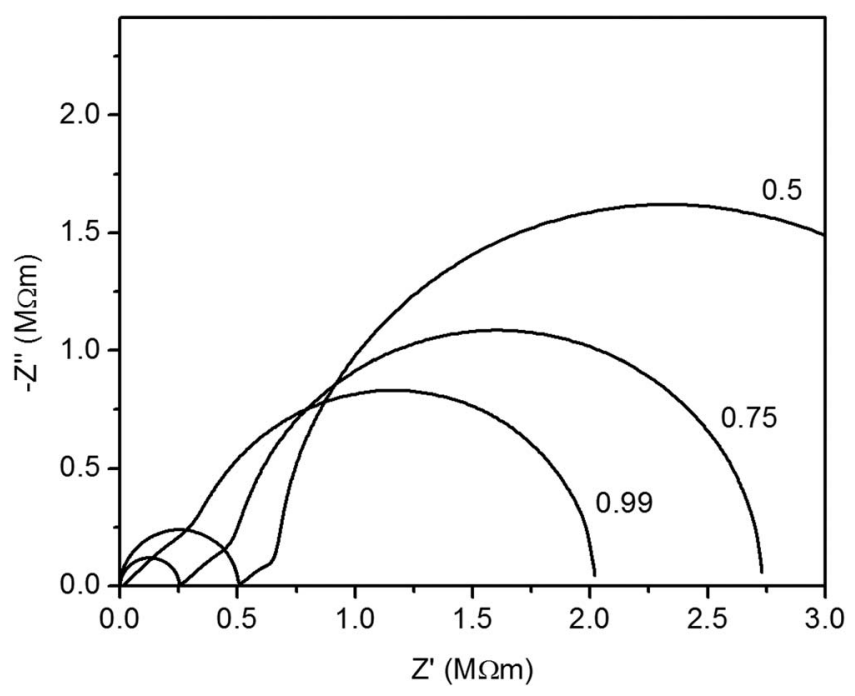

Figure 12. Impedance spectra for the three indicated values of $L_{\text {ode }} / L_{y t e}$ (i.e., the ratio of the electrolyte surface that is covered by the electrode).

the intermediate frequency arc increases in magnitude and the high frequency arc decreases in magnitude as the electrode coverage increases. This result strongly supports the hypothesis that the two arcs arise from processes related to the covered and uncovered portions of the electrolyte, respectively.

Fig. 13 shows the resistance $R_{2}$ as a function of $L_{\text {ode }} / L_{y t e}$ for three different electrolyte aspect ratios. In order to confirm that the absolute size is not important, results are shown for two sets of specimens, one with $L_{y t e}=25 \mu \mathrm{m}$ and one with $L_{y t e}=10 \mu \mathrm{m}$. As before, the results for $R_{2}$ match closely with Eq. 17 when Eq. 19 is satisfied. There is also no observable difference between the large and small samples, again provided Eq. 19 is satisfied.

The effects of varying the exchange current density $J_{0}$ and the electrolyte conductivity $\sigma$ were also studied. Impedance spectra corresponding to several different values of $J_{0}$ are shown in Fig. 14. For small values of $J_{0}$, there is no discernible change in the intermediate frequency arc with exchange current density. However, the intermediate frequency arc begins to vanish along with the low frequency arc

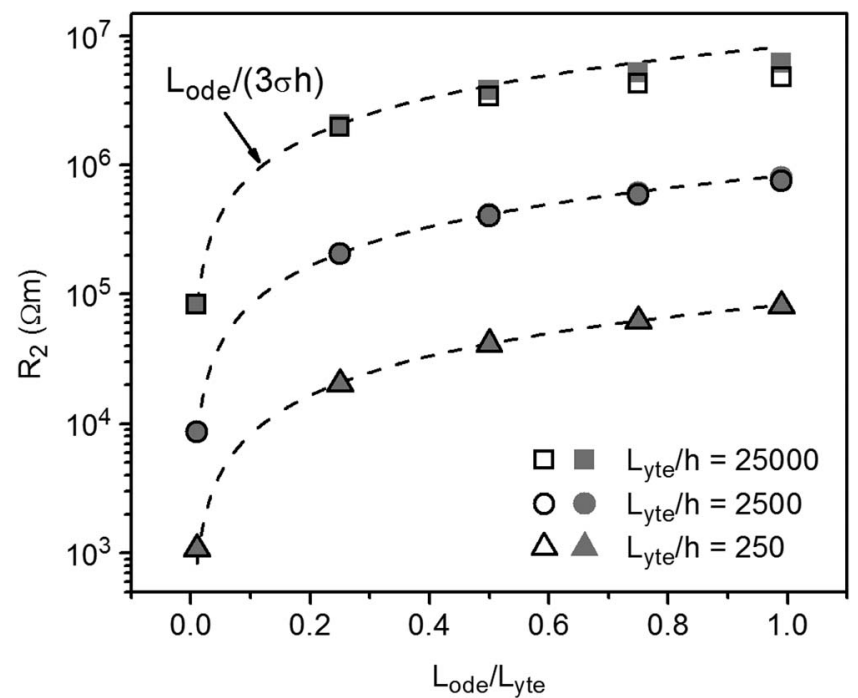

Figure 13. Resistance $R_{2}$ vs. $L_{\text {ode }} / L_{y t e}$ for three different electrolyte aspect ratios, with $L_{y t e}=10 \mu \mathrm{m}$ (filled symbols) and $25 \mu \mathrm{m}$ (open symbols). The semi-empirical linear expression shown is also plotted for each electrolyte aspect ratio as indicated via dashed line.

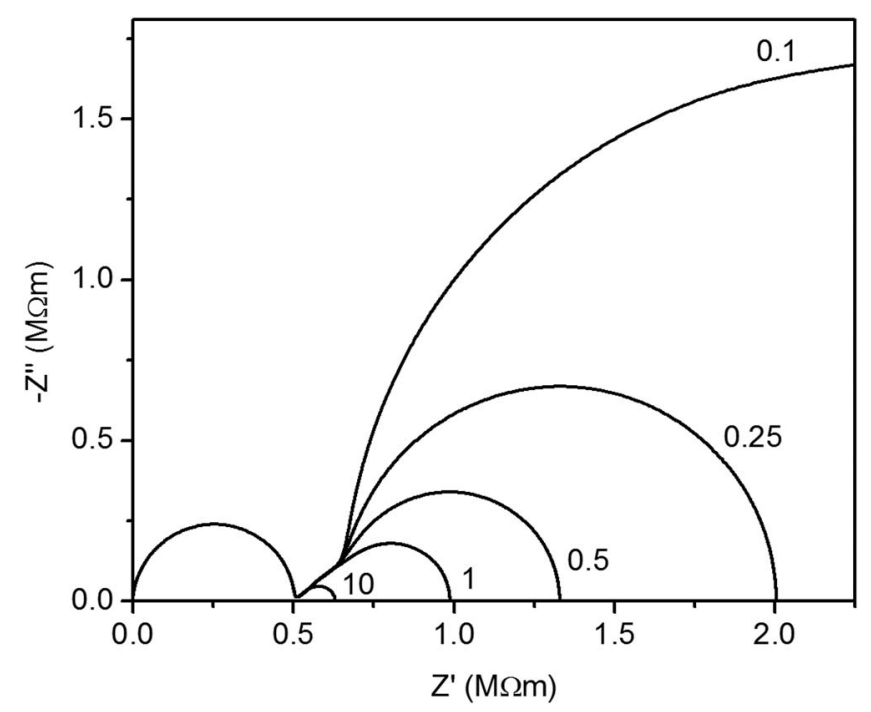

Figure 14. Impedance spectra for specimens with the indicated values of exchange current density in $\mu \mathrm{A} / \mathrm{cm}^{2}$.

as $J_{0}$ is increased, indicating that electrode processes do play a role in the intermediate frequency effect.

Plots of $R_{2}$ as a function of $J_{0}$ are shown in Fig. 15 for a few different values of the electrolyte conductivity $\sigma$. The figure confirms the behavior seen in Fig. 14; the value of $R_{2}$ is independent of $J_{0}$ for small values of $J_{0}$ but decreases for larger values of $J_{0}$. This result is as expected from the previously determined condition Eq. 19, which is not satisfied for large values of $J_{0}$. Indeed, Fig. 15 shows that $R_{2}$ continues to match closely with the semi-empirical expression Eq. 17 when Eq. 19 is satisfied, as indicated by the shaded region of the figure.

Based on the spacing of the curves in Fig. 15, it appears that $R_{2}$ is inversely proportional to the electrolyte conductivity $\sigma$ in the "ideal" regime where the dimensionless quantity $S \gg 1$. This is confirmed by re-plotting the results as a function of resistivity, shown in Fig. 16. A linear relationship Eq. 17 between $R_{2}$ and $1 / \sigma$ is observed when

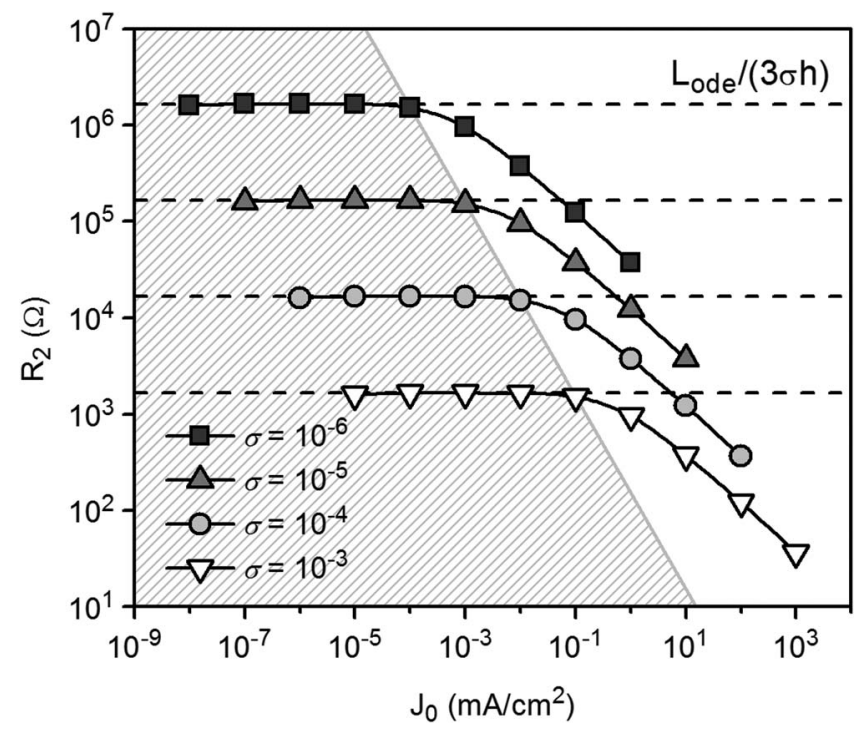

Figure 15. Value of $R_{2}$ as a function of exchange current density $J_{0}$ for various values of electrolyte conductivity in $\mathrm{S} / \mathrm{cm}$. The specimen dimensions are $L_{\text {ode }}$ $=12.5 \mu \mathrm{m}, L_{s}=12.5 \mu \mathrm{m}$, and $h=25 \mathrm{~nm}$. The linear expression for $R_{2}$ shown in the upper right is plotted for each value of $\sigma$, indicated via dashed line. The condition $R_{\text {ode }}>R_{y t e}$ is satisfied within the shaded region. 


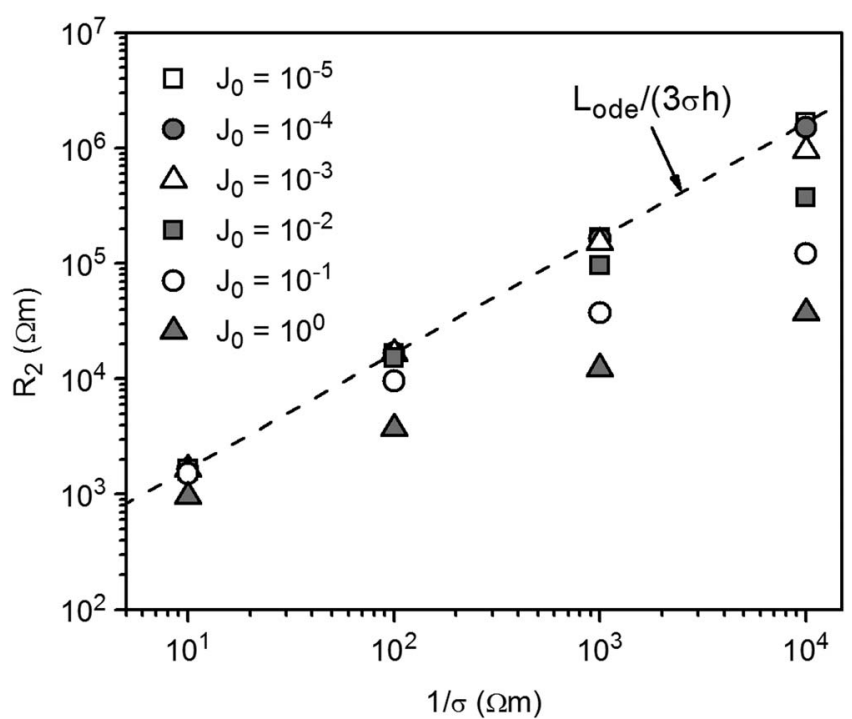

Figure 16. $R_{2}$ as a function of resistivity $1 / \sigma$ for several different values of exchange current density in $\mathrm{mA} / \mathrm{cm}^{2}$. The specimen dimensions are $L_{\text {ode }}=L_{s}$ $=12.5 \mu \mathrm{m}$ and $h=25 \mathrm{~nm}$. The dashed line is a plot of the linear expression for $R_{2}$ indicated.

the relative magnitudes of $J_{0}$ and $\sigma$ are such that $S \gg 1$. Although not evident in the figure, calculation of $S$ at each data point confirmed that when Eq. 19 is not satisfied, a deviation from linearity is observed.

All of the results thus far have demonstrated that so long as Eq. 19 is satisfied, the resistance of the intermediate frequency arc correlates closely with Eq. 17. The form of Eq. 17 seems intuitive as it resembles the theoretical resistance of the portion of the electrolyte under the electrode (region $A B E F$ in Fig. 1). However, the constant factor of $1 / 3$ (hereafter denoted $K$ ) that appears in the expression is more difficult to interpret, and it is still unclear if the value of $K$ depends on the sample geometry. This was investigated more thoroughly using a DC model of the electrolyte geometry with a uniform current distribution at the electrode-electrolyte interface (corresponding to the case where $S \rightarrow \infty$ ). The value of $K$ was computed for different electrolyte aspect ratios and electrode finger geometries, as shown in Fig. 17. As can

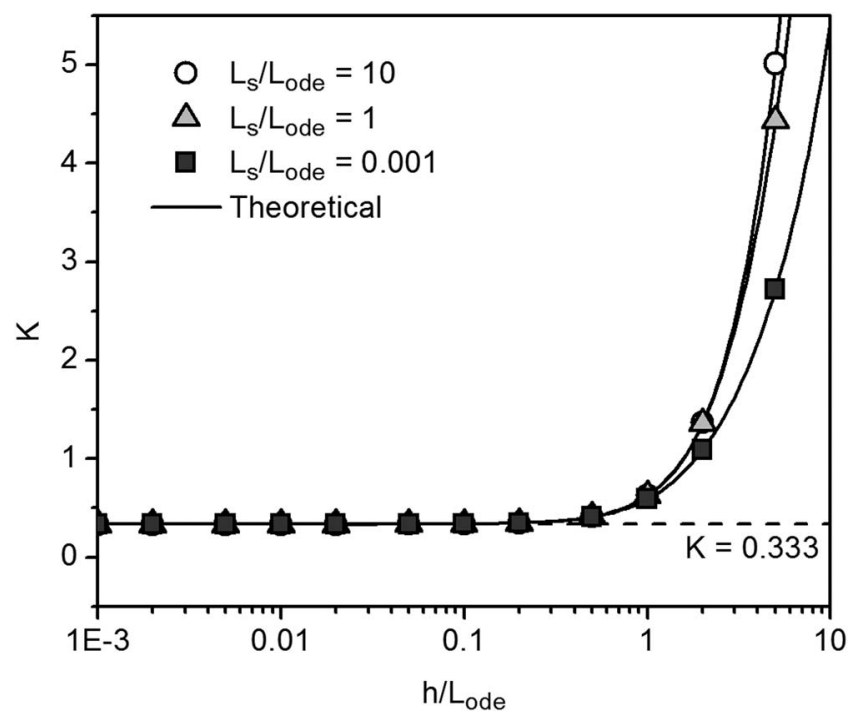

Figure 17. Resistance scaling factor $K$ as a function of aspect ratio $h / L_{o d e}$ for the case where $R_{\text {ode }} \gg R_{\text {yte }}$. Results are shown for three different values of the electrode spacing ratio $L_{s} / L_{\text {ode }}$. Also shown are plots of the same quantity calculated using Eqs. 20 and 21. The value $K=1 / 3$ is indicated by a dashed line.

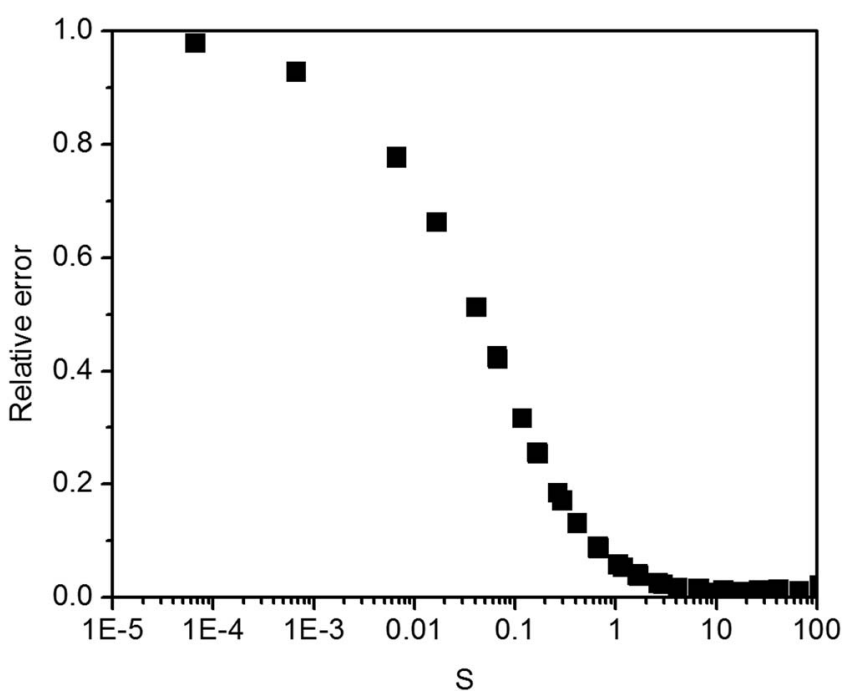

Figure 18. Percent error in intermediate frequency resistance $\left(R_{2}\right)$ estimate calculated using Eq. 17 as a function of the dimensionless number $S$.

be seen, $K$ is approximately equal to $1 / 3$ when the geometric ratio $h /$ $L_{\text {ode }}$ is less than about 0.2 (i.e. the electrode finger width is more than 5 times the electrolyte thickness). For larger values of $h / L_{o d e}$, the value of $K$ increases dramatically. This result seems unintuitive at first glance as it implies that the expected inverse proportionality between resistance and $h / L_{\text {ode }}$ breaks down for thick electrolytes. However, the geometry of the electrodes necessitates a majority of the current remaining near the electrolyte surface, so increasing the electrolyte thickness above a certain value will have a negligible effect on the resistance. Fortunately for the experimenter, many practical thin film devices have $h / L_{\text {ode }}<0.2$, so Eq. 17 is expected to remain broadly applicable.

Thus far, Eq. 19 has been repeatedly asserted as a necessary condition for the validity of approximation Eq. 17, but there has been little quantitative justification. This is clarified in Fig. 18, which plots the relative error in $R_{2}$ calculated using Eq. 17 compared to the observed intermediate frequency resistance against the dimensionless quantity $S$ for all samples considered in the previous sections. According to Eq. 19, this error should be negligible when $S \gg 1$. This is indeed confirmed by the figure, and it is evident that $S \geq 10$ is sufficiently large to achieve an excellent estimate. Even when $S=1$, Eq. 17 gives an error of only about $5 \%$.

It has been established that the intermediate frequency resistance $R_{2}$ can be estimated accurately from the semi-empirical expression Eq. 17 in almost any situation where the condition $S \geq 10$ is met. Additionally, it has been observed that $R_{2}$ tends to zero when $R_{y t e} \gg$ $R_{\text {ode }}$. However, calculating $R_{2}$ in the intermediate regime where $R_{y t e}$ is the same order or slightly larger than $R_{\text {ode }}$ is less straightforward. The DC resistance of geometries similar to Fig. 1 was previously studied analytically by Zhang et al. ${ }^{21}$ From this work, the expected theoretical spreading resistance may be derived as

$$
R_{\text {spread,theo }}=\frac{\bar{R}_{C}}{2 \pi w \sigma}
$$

where $\bar{R}_{C}$ is a function of the relative conductivities of the electrode and electrolyte as well as the geometric parameters. The analytical expression for $\bar{R}_{C}$ is unwieldy, but in the limit of $R_{\text {ode }} / R_{y t e} \rightarrow \infty$ it may be simplified ${ }^{21}$ as

$$
\begin{aligned}
\bar{R}_{C}= & 4 \sum_{n=1}^{\infty} \frac{\operatorname{coth}\left[(n-1 / 2) \pi h / L_{y t e}\right] \sin ^{2}\left[(n-1 / 2) \pi L_{\text {ode }} / L_{y t e}\right]}{(n-1 / 2)^{3}\left(\pi L_{\text {ode }} / L_{y t e}\right)^{2}} \\
& -\frac{2 \pi\left(L_{y t e}-L_{\text {ode }}\right)}{h}
\end{aligned}
$$


Thus if Eq. 19 is satisfied, it is expected that the theoretical value of $R_{2}$ can be calculated using Eqs. 20 and 21. This result bears little resemblance to the expression Eq. 17 which was deduced from the parametric study, but the two expressions do in fact yield nearly identical results in the range of parameter values of interest. Values of the scaling factor $K$ calculated from Eq. 20, shown as solid lines in Fig. 17, agree closely with the results of the DC modeling. Thus when $S \geq 10$ and $L_{\text {ode }}>5 h, R_{2}$ can be estimated to a high degree of accuracy using Eq. 17. Unfortunately a general closed-form expression for $K$ could not be determined, so very thick films with $L_{\text {ode }}<5 h$ require the use of Eq. 20 to calculate $R_{2}$. When the condition $S \gg 1$ is satisfied, $\bar{R}_{C}$ can be calculated from Eq. 21 ; otherwise, the general expression given in Appendix A of Ref. 21 must be used.

Comparison to equivalent circuit models. - With the physical meaning of the intermediate frequency impedance now better understood, the question arises of whether, for experimental fitting purposes, it might be simpler to replace the relatively complex model described here with a simpler equivalent circuit model. In order to investigate further, equivalent circuit fitting of the spectra shown in Fig. 10 was performed using ZView (SAI, v.3.2d). The fitting used the equivalent circuit shown in Fig. 2, whose adjustable parameters include the three resistance values $R_{l}, R_{2}$, and $R_{3}$ corresponding to the high, intermediate, and low frequency arcs, respectively. In order for the equivalent circuit model to be useful, these values should correspond to physically meaningful properties of the electrochemical cell. For $h=25$ $\mathrm{nm}$, the equivalent circuit fit yields a value of $R_{3}=3.31 \mathrm{M} \Omega$ corresponding to the low frequency arc. Using Eq. 15 it is possible to calculate $J_{0}=0.101 \mu \mathrm{A} / \mathrm{cm}^{2}$, an error of approximately $1 \%$ from the actual value of $0.1 \mu \mathrm{A} / \mathrm{cm}^{2}$. The other two resistances obtained from the equivalent circuit, $R_{l}=505 \mathrm{k} \Omega$ and $R_{2}=179 \mathrm{k} \Omega$, can be used to independently quantify $\sigma$ using Eqs. 13 and 17 respectively. From $R_{l}$ a value of $\sigma=0.990 \mathrm{mS} / \mathrm{m}$ is obtained, an error of less than $1 \%$ from the true value, $1 \mathrm{mS} / \mathrm{m}$. Calculating the conductivity from $R_{2}$ instead yields $\sigma=0.932 \mathrm{mS} / \mathrm{m}$, which is an error of approximately $7 \%$. Since Eq. 17 is an empirical, approximate relation, it is unsurprising that it gives a less accurate value of $\sigma$. Nonetheless, the equivalent circuit in this case is demonstrably useful in quantifying the system parameters, given the equations derived in this work to explain the physical meaning of the elements.

On the other hand, it was noted earlier that impedance spectra for very thin electrolytes could yield misleading results when fitted with an equivalent circuit, as the intermediate frequency arc becomes large enough to alter the apparent magnitude of the electrode arc. For $h=$ $1 \mathrm{~nm}$, the equivalent circuit gives $R_{3}=4.79 \mathrm{M} \Omega$, which is clearly problematic since with only the electrolyte thickness having changed, the electrode resistance is expected to be the same as in the previous case. Calculating the exchange current density yields $J_{0}=0.070$ $\mu \mathrm{A} / \mathrm{cm}^{2}$, which differs from the actual value by $30 \%$. This overestimation of electrode polarization resistance reveals a notable deficiency of an equivalent circuit model as applied to thin film geometries, and caution should be exercised when applying such models generally.

\section{Conclusions}

An impedance model of a thin film electrochemical cell incorporating a phenomenological description of the electrode-electrolyte interface was developed. The model was able to fit complex experimental impedance spectra for Pt/YSZ and Pt/GDC cells using only four adjustable parameters. This represents a substantial improvement over comparable models based on equivalent circuits, which require up to nine fitting parameters. Further, the new model was shown to be able to quantify electrode parameters more accurately than an equivalent circuit in the case of very thin films. Comparison of the fitting parameter values to literature provided evidence of the validity of the model. However, due to the sample geometry, the ability to accurately measure the permittivity of thin film electrolytes has been shown to be limited.
A parametric study using the new model enabled the mechanism of a previously unexplained intermediate frequency impedance arc to be understood. The arc arises from spreading of current at low frequencies due to the high resistance of the electrode-electrolyte interface. At higher frequencies, the impedance of the interface is reduced by the capacitive displacement currents, allowing a shorter current path through the electrolyte. The magnitude of the spreading resistance was quantified and matched the expected theoretical value. In addition, a much simpler estimate of the spreading resistance was shown to be valid in many cases.

\section{References}

1. J. E. Bauerle, J. Phys. Chem. Solids, 30, 2657 (1969).

2. J. R. MacDonald (Ed.), Impedance spectroscopy: Emphasizing solid materials and systems, John Wiley \& Sons, New York (1987).

3. J. T. S. Irvine, D. C. Sinclair, and A. R. West, Adv. Mater, 2, 132 (1990).

4. J. Jamnik and J. Maier, Phys. Chem. Chem. Phys., 3, 1668 (2001).

5. D. A. Harrington and P. van den Driessche, Electrochim. Acta, 56, 8005 (2011).

6. W. G. Bessler, Solid State Ionics, 176, 997 (2005).

7. S. B. Adler, B. T. Henderson, M. A. Wilson, D. M. Taylor, and R. E. Richards, Solid State Ionics, 134, 35 (2000).

8. S. B. Adler, J. Electrochem. Soc., 149, E166 (2002).

9. A. Bieberle and L. J. Gauckler, Solid State Ionics, 146, 23 (2002).

10. S. Sunde, J. Electroceramics, 5, 153 (2000).

11. J. Fleig, Solid State Ionics, 161, 279 (2003).

12. A. Bieberle, L. P. Meier, and L. J. Gauckler, J. Electrochem. Soc., 148, A646 (2001).

13. R. Radhakrishnan, A. V. Virkar, and S. C. Singhal, J. Electrochem. Soc., 152, A210 (2005).

14. R. Radhakrishnan, A. V. Virkar, and S. C. Singhal, J. Electrochem. Soc., 152, A927 (2005).

15. L. A. Dunyushkina, Y. Lu, and S. B. Adler, J. Electrochem. Soc., 152, A1668 (2005).

16. A. K. Opitz and J. Fleig, Solid State Ionics, 181, 684 (2010).

17. J. L. Hertz and H. L. Tuller, J. Electroceramics, 13, 663 (2004)

18. J. L. Hertz and H. L. Tuller, Solid State Ionics, 178, 915 (2007).

19. J. Jiang, W. Shen, and J. L. Hertz, Solid State Ionics, 249-250, 139 (2013).

20. R. S. Timsit, IEEE Trans. Components Packag. Technol., 33, 636 (2010).

21. P. Zhang, Y. Y. Lau, and R. M. Gilgenbach, J. Appl. Phys., 109, 124910 (2011).

22. L. R. Velho and R. W. Bartlett, Metall. Trans., 3, 65 (1972).

23. A. Jaccoud, C. Falgairette, G. Fóti, and C. Comninellis, Electrochim. Acta, 52, 7927 (2007).

24. R. Schmiedl, V. Demuth, P. Lahnor, H. Godehardt, Y. Bodschwinna, C. Harder, L. Hammer, H.-P. Strunk, M. Schulz, and K. Heinz, Appl. Phys. A Mater. Sci. Process., 62, 223 (1996).

25. J. Fleig and J. Maier, J. Electroceramics, 1, 73 (1997).

26. B. E. McNealy and J. L. Hertz, Solid State Ionics, 256, 52 (2014).

27. S. B. Adler, Chem. Rev., 104, 4791 (2004).

28. D. Y. Wang and A. S. Nowick, J. Electrochem. Soc., 126, 1155 (1979).

29. M. J. Verkerk, M. W. J. Hammink, and A. J. Burggraaf, J. Electrochem. Soc., 130, 70 (1983).

30. J. Mizusaki, K. Amano, S. Yamauchi, and K. Fueki, Solid State Ionics, 22, 313 (1987).

31. W. Schmickler and E. Santos, Interfacial Electrochemistry, 2nd ed., p. 94, Springer, Heidelberg (2010)

32. N. L. Robertson and J. N. Michaels, J. Electrochem. Soc., 138, 1494 (1991)

33. J. Jiang and J. L. Hertz, J. Electroceramics, 32, 37 (2013).

34. G. Chiodelli, L. Malavasi, V. Massarotti, P. Mustarelli, and E. Quartarone, Solid State Ionics, 176, 1505 (2005).

35. J. H. Joo and G. M. Choi, J. Eur. Ceram. Soc., 27, 4273 (2007).

36. D. Pérez-Coll, P. Núñez, J. R. Frade, and J. C. C. Abrantes, Electrochim. Acta, 48, 1551 (2003)

37. I. Kosacki, C. M. Rouleau, P. F. Becher, J. Bentley, and D. H. Lowndes, Electrochem. Solid-State Lett., 7, A459 (2004).

38. A. Karthikeyan, C.-L. Chang, and S. Ramanathan, Appl. Phys. Lett., 89, 183116 (2006).

39. M. Sillassen, P. Eklund, N. Pryds, E. Johnson, U. Helmersson, and J. Bøttiger, $A d v$ Funct. Mater, 20, 2071 (2010).

40. J. Jiang, X. Hu, W. Shen, C. Ni, and J. L. Hertz, Appl. Phys. Lett., 102, 143901 (2013).

41. T. Suzuki, I. Kosacki, and H. U. Anderson, Solid State Ionics, 151, 111 (2002).

42. L. Chen, C. L. Chen, X. Chen, W. Donner, S. W. Liu, Y. Lin, D. X. Huang, and A. J. Jacobson, Appl. Phys. Lett., 83, 4737 (2003).

43. A. J. A. Winnubst, A. H. A. Scharenborg, and A. J. Burggraaf, Solid State Ionics, 14, 319 (1984).

44. J. Mizusaki, K. Amano, S. Yamauchi, and K. Fueki, in Chemical sensors, T. Seiyama, Editor, p. 277, Elsevier, Amsterdam (1983).

45. R. Hartung, Z. Phys. Chem. (Leipzig), 260, 259 (1979).

46. J. Fontanella and R. L. Johnston, J. Non. Cryst. Solids, 31, 401 (1979)

47. J. Fontanella, C. Andeen, and D. Schuele, J. Appl. Phys., 45, 2852 (1974).

48. A. K. Harman, S. Ninomiya, and S. Adachi, J. Appl. Phys., 76, 8032 (1994)

49. P. Zhang, Y. Y. Lau, and R. S. Timsit, IEEE Trans. Electron Devices, 59, 1936 (2012). 